\title{
Haploidization Analysis in Penicillium chrysogenum
}

\author{
By C. BALL* \\ Glaxo Laboratories Limited, Ulverston, Lancashire
}

(Accepted for publication 22 February 1971)

\begin{abstract}
SUMMARY
Results relevant to the problem of increasing penicillin yield in Penicillium chrysogenum by recombination through the parasexual cycle are discussed. Sister strains differing from each other in few mutational steps have been used to construct a map with three haploidization groups. By this approach it has been possible to overcome those barriers to recombination, principally chromosome rearrangements, which arise when non-sister strains are used. Selection against an allele has been demonstrated and the problem solved by use of $p$-fluorophenylalanine to induce haploidization. In addition, the selection of a morphologically stable strain from an unstable strain has been achieved and the genetic determinant for this instability allocated to a haploidization group.
\end{abstract}

\section{INTRODUCTION}

In the imperfect fungus Penicillium chrysogenum initial improvements for penicillin yield depended upon mutation and selection (Elander, 1966). Discovery of the parasexual cycle and its occurrence in asexual fungi offered hope of breeding for improved penicillin yield (Pontecorvo \& Sermonti, I954; Pontecorvo, 1956). In the first attempts (Sermonti, 1959) the only strain with improved titre was a diploid. Macdonald and co-workers (Macdonald, Hutchinson \& Gillet, 1964, I965; Macdonald, 1968) met two main difficulties. These were the possible selection against segregants of high titre and 'parental genome segregation' in which the haploids of parental type were preferentially recovered. The latter was probably due to the use of parent haploids which differed from each other in chromosomal rearrangements; for example, a reciprocal translocation in one parent would prevent recombination at haploidization between the linkage groups concerned.

The essential aim in this study was to try to overcome the problems that had been met in haploidization. Success in this would permit the building of linkage maps which could provide a basis for a more rational planned programme of breeding. The most promising approach appeared to be through the use of sister strains, differing from each other in only a few mutational steps and, if possible, the use of $p$-fluorophenylalanine (PFA) as an agent to assist in the production of segregant haploids (Lhoas, 196I; Morpurgo, 196I).

\section{METHODS}

The media, complete medium (CM) and minimal medium (MM) and methods used were identical to those of Macdonald, Hutchinson \& Gillett (1963a,b,c), with the exception that nucleic acid hydrolysates were not added to the CM. The following methods differed from those included in this earlier work.

Haploid and diploid recognition. Heteroallelic diploids were distinguished initially from heterokaryons and parental haploid strains on the basis of spore colour and prototrophy.

\footnotetext{
* Present address: Glaxo Research Ltd, Stoke Poges, Buckinghamshire
} 
However, the criterion of spore size (Pontecorvo \& Sermonti, I954) is essential for the classification of segregants. In the present work, diploids had a larger mean spore diameter $(5 \cdot 4 \mu \mathrm{m}$.) than haploids ( $4 \mu \mathrm{m}$.) and were usually less variable.

Haploidization analysis. A modified version of the PFA technique used with Aspergillus was employed. In order to minimize the possible selection by PFA against certain alleles, colonies were transferred from PFA medium to PFA-free medium. An inhibitory concentration of PFA $(0.02 \mathrm{M})$ was used such that $10 \%$ of the diploid spores survived. These conditions were found to be optimal for the recovery of a high frequency of haploid segregants as sectors, provided the smallest colonies were subcultured from PFA after 4 days incubation. While the sectors produced were probably induced by PFA, haploid segregants existing in the treated population could be selected by PFA treatment. However, the latter arose as whole colonies and were avoided in analysis.

Mutants. The symbols used for the u.v.-induced mutants referred to in this work were as follows: $w I, w 2$ (white spore colour); $b r I, b r 2$ (brown spore colour); $y I, y 2$ (yellow spore colour); $b g I, b g 2$ (bright green spore colour); chor, choz (requirement for choline); lysI, lys2, lys3, lys4 (requirement for lysine); anr (requirement for aneurin); thior (requirement for thiosulphate); hisI (requirement for histidine); nicr (requirement for nicotinamide); $r i b r$ (inability to grow on ribose as sole carbon source); $a a$ (resistance to 8 aza-adenine). Ultraviolet light was chosen as the mutagen since previous studies (Käfer \& Chen, I964; Azevedo \& Roper, 1967 ) had indicated that at I to $5 \%$ survival translocations did not occur in high frequency in Aspergillus nidulans. In expressing the genetic constitution of a strain semi-colons are used to distinguish between genes on different haploidization groups.

\section{RESULTS}

The morphologically unstable strain initially selected for study was derived from Q1 76 by serial irradiation and selection for increased penicillin yield. Like QI 76 (Stauffer \& Backus, 1954) conidia of this strain when plated onto CM produced colonies of varied growth rate and conidiation due to genetic instability of the strain. About $5 \%$ of these colonies had slow growth and poor conidiation, the rest had faster growth rate and denser conidiation. Both classes were relatively stable on further plating but each again produced a proportion (about $5 \%$ ) of the alternative type. However, three generations of selection for faster growth rate and denser sporulation gave a more stable line. Colonies produced from spore platings of the latter appeared identical; therefore it was used as the parent strain for marker induction and subsequent genetic analysis.

\section{Genetic analysis}

Spore colour complementation. Several independently isolated spore-colour mutants were tested in pairs for complementation in heterokaryons and diploids. In the diploid all brown, yellow and bright green mutants complemented with white spore-colour mutants in that the dark green wild-type spore colour was produced. Mutants of like colour did not complement. Complementation was also shown by the following combinations in the diploid: $b r I / y r$, bgI/brI and $b g 2 / b r 2$.

No complementation was shown by $b r 2 / y I, b g I / y I$ and $b g 2 / y I$ and thus it was concluded that spore-colour mutants other than white were mutants of adjacent regions of the genome or at least are related functionally.

Autonomous spore colour was generally observed in heterokaryons with the exception of combinations $y I$ with $b r I$ and $w I$ with $b r I$. In these cases heterokaryons were dark green. 
Linkage. In Aspergillus nidulans haploids are derived from diploids as a result of segregation of whole chromosomes largely without crossing over. Coincidence of mitotic crossing over is rare (Pontecorvo \& Käfer, I958). However, in Verticillium albo-atrum (Hastie, I967) and Aspergillus niger (Lhoas, 1967) a high frequency of mitotic crossing over makes such coincidence much more probable. Haploidization groups can only be considered as approximations to linkage groups since no meiotic confirmation of inferred linkage is possible. In Penicillium chrysogenum the same principles apply. Thus criteria of linkage and non-linkage for any two loci employed in this work have been two- or four-class segregation respectively.

Table I. Classification of haploid segregants derived from two diploids after treatment with $P F A^{*}$

Diploid: wI chor/brI; lysI

WI $\left\{\begin{array}{l}\text { chor } \\ \text { chor }\end{array}\right.$
brI $\left\{\begin{array}{l}\text { chor } \\ \text { choI }\end{array}\right.$
Totals
Tor

Diploid: yI chor/brI; lysI

lys $I$

lys

ys $I^{+}$
I0
0
0
6
16

$$
\begin{gathered}
\text { yI }\left\{\begin{array}{l}
\text { chor } \\
\text { chor }
\end{array}\right. \\
\text { brI }\left\{\begin{array}{l}
\text { chor } \\
\text { chor }
\end{array}\right.
\end{gathered}
$$

Totals

lys $I$
14
0
0
3
17

17

\begin{tabular}{|c|c|}
\hline Subtotals & Totals \\
\hline $\begin{array}{r}18 \\
0\end{array}$ & I 8 \\
\hline 0 & I 5 \\
\hline I5 & \\
\hline
\end{tabular}

lys $I^{+}$
6
0
0
14
20

\begin{tabular}{|c|c|}
\hline Subto & Totals \\
\hline 20 & 20 \\
\hline 0 & \\
\hline 0 & I7 \\
\hline 17 & $1 /$ \\
\hline
\end{tabular}

* Results show linkage of $w I, b r I, y I$ and choI and independent segregation of lysI.

In the former case only the two parental genotypes were recovered in approximately equal frequency, and in the latter case two parental and two recombinant genotypes were recovered, each of the four classes being recovered in approximately the same frequency. Recovery of only one recombinant class in high frequency was considered equivocal for mapping purposes.

The diploids used in defining the existence of at least three haploidization groups in Penicillium chrysogenum were as follows: WI choI/brI; lysI (Tables I and 2); yI chor/brI; lysI (Tables I and 2); wI choI; hisI/br; lysI (Table 3); brI; hisI; lysI/wI; nicI (Table 3).

In each case the diploid was dark green, differing slightly in shade from the original green haploid strain from which the components were produced. Thus if normal dark green haploid segregants had been produced they could have been detected. Since none was found, the spore-colour mutants were allocated to the same haploidization group. Linkage to the marker chor (Table I) substantiates these conclusions.

Table 2 shows the results of analysis of spontaneous haploid segregants from diploids which were later subjected to PFA treatment (see Table I). A comparison of Tables I and 2 clearly shows that the lysi allele confers a selective growth advantage to segregants. Only in the presence of PFA is the dominant allele of lysI recovered. This is probably achieved because the segregant sectors which arose following PFA treatment came from very poor growing colonies presumed to be aneuploid (see below).

Subsequent analysis has permitted allocation of more than 20 independently isolated mutants to one or other of three haploidization groups (Tables 3 and 4 ). Included in this is a determinant for unstable morphology ' $U S$ ' which was allocated to group III. 
The ' $U S$ ' determinant was dominant in that the diploid lys4; ' $U S$ ' $/$ brI; hisI; nicI exhibited all the characteristics of the unstable parent. A stable derivative of this unstable diploid was selected. This gave segregants that were morphologically normal. It was concluded that the transition from unstable to stable had been accomplished by either mutation of the ' $U S$ ' determinant or mitotic crossing over or non-disjunction to give homozygosity for the stability locus.

Table 2. Classification of haploid segregants derived from two diploids as sectors, without PFA-treatment

Diploid: wI chor/brI; lyst

Diploid: yI chor/brI ; lysI

$$
\begin{aligned}
& \text { wI }\left\{\begin{array}{l}
\text { chor } \\
\text { chor }
\end{array}\right. \\
& \text { brI }\left\{\begin{array}{l}
\text { chor } \\
\text { choI }
\end{array}\right.
\end{aligned}
$$

Totals

$$
\begin{gathered}
\text { lysI } \\
\text { I I } \\
0 \\
0 \\
\text { I I } \\
22
\end{gathered}
$$

lysI

17

$$
\begin{gathered}
\text { yI }\left\{\begin{array}{l}
\text { chor } \\
\text { chor }
\end{array}\right. \\
\text { brI }\left\{\begin{array}{l}
\text { chor } \\
\text { choI }
\end{array}\right.
\end{gathered}
$$

\begin{tabular}{|c|c|c|}
\hline lysI $I^{+}$ & Subtotals & Totals \\
\hline $\begin{array}{l}0 \\
0\end{array}$ & $\begin{array}{r}17 \\
0\end{array}$ & 17 \\
\hline 0 & I & 14 \\
\hline I & I3 & \\
\hline
\end{tabular}

Totals

lysi
$\mathbf{I}$
0
0
0
$\mathrm{I}$

Subtotals

Totals

$$
\begin{aligned}
& \left.\begin{array}{r}
12 \\
0
\end{array}\right\} \quad I 2 \\
& \left.\begin{array}{r}
0 \\
\text { II }
\end{array}\right\} \text { II }
\end{aligned}
$$

Table 3. Classification of haploid segregants from two PFA-treated diploids showing free recombination between three haploidization groups*

Diploid: wI choI; hisI/brI; lysI

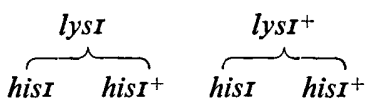

Subtotals Totals

$$
\begin{gathered}
\text { wI }\left\{\begin{array}{l}
\text { chor } \\
\text { choI }
\end{array}\right. \\
\text { brI }\left\{\begin{array}{l}
\text { chor } \\
\text { choI }
\end{array}\right. \\
\text { Subtotals } \\
\text { Totals }
\end{gathered}
$$

\begin{tabular}{|c|c|c|c|}
\hline $\begin{array}{l}\mathbf{I} \\
0\end{array}$ & 0 & $\begin{array}{l}4 \\
6\end{array}$ & \} 10 \\
\hline 0 & $\begin{array}{l}\text { I } \\
\text { O }\end{array}$ & $\begin{array}{l}2 \\
6\end{array}$ & 8 \\
\hline 2 & 4 & & \\
\hline
\end{tabular}
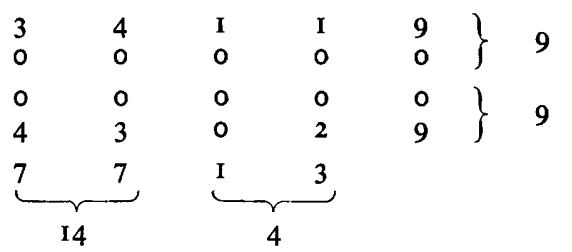

Diploid: brI; hisI ; lysI/wI; nicr
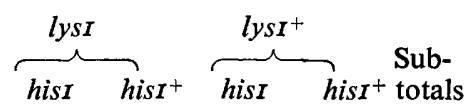

$$
\begin{gathered}
\text { wI }\left\{\begin{array}{l}
\text { nicI } \\
\text { nicI }
\end{array}\right. \\
\text { brI }\left\{\begin{array}{l}
\text { nicI } \\
\text { nicI }
\end{array}\right. \\
\text { Subtotals } \\
\text { Totals }
\end{gathered}
$$$$
0
$$

$\begin{array}{ll}\begin{array}{ll}0 & 0 \\ 2 & 4 \\ 0 & 0 \\ 2 & 4 \\ 4 & 8\end{array} & \begin{array}{ll}42 & \end{array}\end{array}$

${ }^{*} w I$ and $b r I$ are linked, as are lysI and nicr. Both these groups freely recombine. hisI freely recombines with both these groups.

\section{Mechanisms of segregation}

Haploidization. A slow growing, dark green prototrophic colony derived from PFA treatment of diploid $y I$ chor/brI; lysI was found to produce faster growing normal sectors. The genotypes and ploidy of such sectors were classified at each of three plating generations 
of slow-growing prototrophs. In the first generation haploids of genotypes bri and yI choI and diploids of phenotype $y I$ chor and $y I$ chor; lysI were recovered. In the second generation haploids of genotype brI; lySI and yI chor; lysI and diploids of phenotype yI chor were detected. In the final generation similar types to those found for the second generation were recovered. In addition, at each generation dark green prototrophic diploids were found.

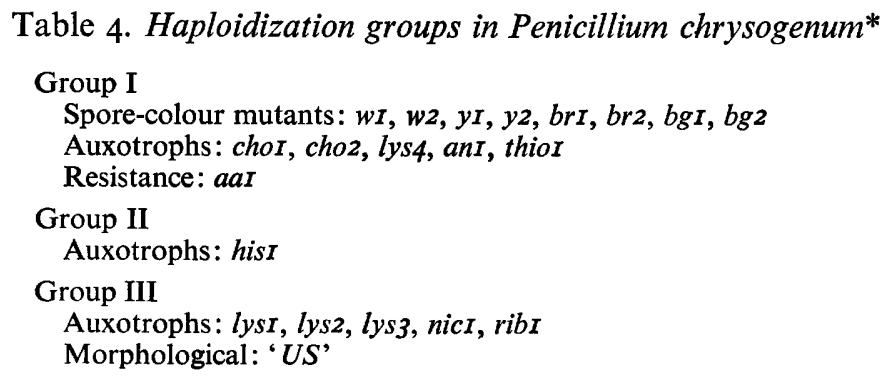

* See text for description of allele symbols. Of mutants of like type in any one group, only the sporecolour mutants have been subjected to complementation tests.

It was concluded that the slow-growing prototrophic colony, while being heterozygous for all the known loci, was probably a $2 n-\mathrm{I}$ aneuploid being monosomic for an unmarked linkage group. This conclusion was reached because the patterns of segregation were very similar to those found by Käfer (I96I) when non-disjunction and chromosome loss were postulated as causes of haploidization in Aspergillus nidulans.

Mitotic crossing-over. Suspensions of conidia of the dark green (wild-type spore colour) diploid yI chor/bri; lysI were treated with a dose of u.v. that gave $50 \%$ survival when plated onto CM. A proportion of the resulting colonies were mosaic for conidial colour. Five such mosaics were analysed. Two were a mixture of yellow and brown prototrophic diploids and three a mixture of yellow, brown and dark green diploid prototrophs. Since $y I$ and chor are linked (Tables I and 4) non-disjunction could not give yellow prototrophs. Therefore it is likely that mitotic crossing over had occurred. Indeed, the yellow and brown prototrophs contained in these mosaic colonies probably represent the reciprocal products of a single mitotic crossing-over event. In addition, it was possible to detect dark green chor homozygotes and therefore it was concluded that $y_{I}$ and chor mark different arms of group I. The following diagram illustrates the proposed distribution of markers at the four-strand stage of mitotic division.

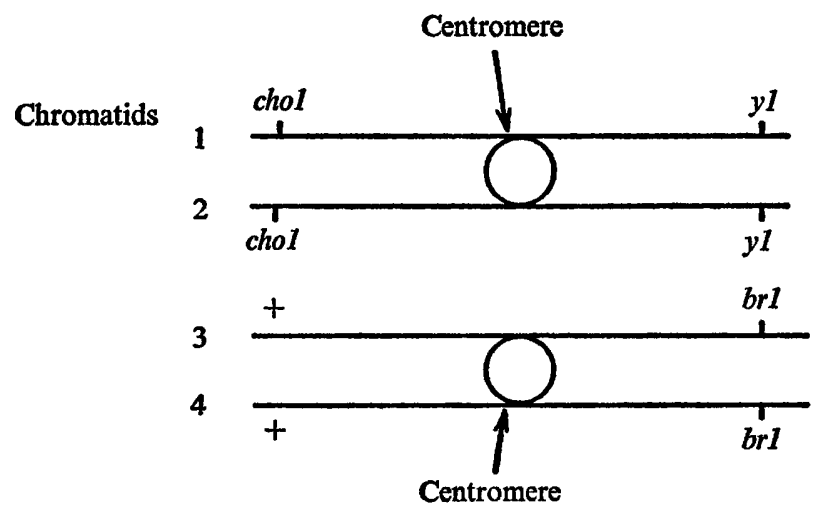


A single crossover between the centromere and the spore colour markers gives reciprocal products of segregation with genetic constitution $y_{I}$ chor $/ y I$ (chromatid I with 3 ) and brI chor/bri (chromatid 2 with 4). Subsequent PFA breakdown of certain of these yellow and brown prototrophs confirmed that they were heterozygous for chor.

From the frequency of yellow prototrophs arising spontaneously as sectors from the centre of normal diploid colonies ( $I$ in 300 ) it was possible to estimate the frequency of mitotic crossing-over for the whole genome (six chromosome arms) at $4 \%$ (I2/300 $\times 100)$. This value is approximately 20 times higher than that found for Aspergillus nidulans (Pontecorvo \& Käfer, 1958) and approximately five times less than that found in A. niger (Lhoas, I967).

\section{DISCUSSION}

This study has shown that problems in undertaking the formal genetic analysis of Penicillium chrysogenum through the parasexual cycle can be surmounted. The use of relatively stable sister strains in conjunction with haploidization induced by PFA has enabled two major problems posed in earlier studies to be overcome, namely 'parental genome segregation' and possible selection against alleles.

The way is now open for allocation of a number of loci, which increase penicillin yield, to their haploidization groups. Subsequently, such increases can be combined in a controlled way either by haploidization or mitotic crossing-over to produce desired combinations of alleles.

Recent studies suggest that this has been achieved by haploidization (Ball, I970). However, it should be possible to recombine genes on the same haploidization group in a controlled way, using mitotic crossing-over by use of heterozygous recessive selective markers such as spore-colour mutants. Yield-increasing loci distal to such markers should become homozygous, thus enabling control to be exerted over the breeding process. An important aspect of this is that more recessive selective markers (e.g. drug resistance) need to be produced.

In addition, the present work has shown that a morphologically unstable determinant, analogous to that described by Stauffer \& Backus (1954), has been allocated to a haploidization group. Recent studies with Aspergillus nidulans (Bainbridge \& Roper, 1966; Azevedo \& Roper 1967; Ball, 1967; Nga \& Roper, 1967) have provided mechanisms based on partial chromosome duplication that could well explain the unstable system studied here.

The author wishes to acknowledge the technical assistance of Miss J. A. Weatherburn at all stages of this work.

\section{REFERENCES}

Azevedo, J. A. \& Roper, J. A. (1967). Lethal mutations and balanced lethal systems in Aspergillus nidulans. Journal of General Microbiology 49, 149-155.

BaLl, C. (1967). Chromosome instability related to gene suppression in Aspergillus nidulans. Genetical Research, Cambridge ro, 173-183.

BALL, C. (1970). Improvement of penicillin productivity in Penicillium chrysogenum by recombination. First International Symposium on the Genetics of Industrial Micro-organisms. Prague: Academia (In Press.)

BAINBRIDGe, B. W. \& Roper, J. A. (1966). Observations on the effect of a chromosome duplication in Aspergillus nidulans. Journal of General Microbiology 42, 41 2-424.

Elander, R. P. (I966). Enhanced penicillin biosynthesis in mutant and recombinant strains of Penicillium chrysogenum. Abhandlungen der Deutschen Akademie der Wissenschaften zu Berlin pp. 403-423.

HASTIE, A. C. (1967). Mitotic recombination in conidiophores of Verticillium albo-atrum. Nature, London 214, 249-252. 
KÄFER, E. (1961). The process of spontaneous recombination in vegetative nuclei of Aspergillus nidulans. Genetics 46, 158I-1609.

KÄFER, E. \& CHEN, T. L. (1964). Translocations and recessive lethals induced in Aspergillus by ultraviolet light and gamma-rays. Canadian Journal of Genetics and Cytology 6, 249-254.

LHOAS, P. (196I). Mitotic haploidization by treatment of Aspergillus niger diploids with $p$-fluorophenylalamine. Nature, London I9o, 734.

LHOAs, P. (1967). Genetic analysis by means of the parasexual cycle in Aspergillus niger. Genetical Research, Cambridge 10, 45-6I.

MORPURGO, G. (196I). Somatic segregation induced by $p$-fluorophenylalanine. Aspergillus Newsletter 2, I0.

MACDONALD, K. D. (1968). The persistence of parental genome segregation in Penicillium chrysogenum after nitrogen mustard treatment. Mutation Research 5, 302-305.

Macdonald, K. D., Hutchinson, J. M. \& Gillett, W. A. (I963a). Isolation of auxotrophs of Penicillium chrysogenum and their penicillin yields. Journal of General Microbiology 33, 365-374.

Macdonald, K. D., Hutchinson, J. M. \& Gillett, W. A. (1963b). Heterokaryon studies and the genetic control of penicillin and chrysogenin production in Penicillium chrysogenum. Journal of General Microbiology 33, 375-383.

Macdonald, K. D., Hutchinson, J. M. \& Gillett, W. A. (1963c). Formation and segregation of heterozygous diploids between and wild-type strain and derivatives of high penicillin yield in Penicillium chrysogenum. Journal of General Microbiology 33, 385-394.

Macdonald, K. D., Hutchinson, J. M. \& Gillett, W. A. (1964). Properties of heterozygous diploids between strains of Penicillium chrysogenum selected for high penicillin yield. Antonie van Leeuwenhoek 33, 209-224.

Macdonald, K. D., Hutchinson, J. M. \& Gillett, W. A. (1965). Heterozygous diploids of Penicillium chrysogenum and their segregation patterns. Genetics 36, 378-397.

NGA, B. H. \& Roper, J. A. (1967). Quantitative intrachromosomal changes arising at mitosis in Aspergillus nidulans. Genetics 58, 193-209.

PonteConvo, G. (1956). The parsexual cycle in fungi. Annual Review of Microbiology 1o, 393-400.

PONTECORVo, G. \& KäFER, E. (1958). Genetic analysis by means of mitotic recombination. Advances in Genetics 9, 7I-I04.

Pontecorvo, G. \& Sermonti, G. (1954). Parsexual recombination in Penicillium chrysogenum. Journal of General Microbiology II, 94-104.

SermontI, G. (1959). Genetics of penicillin production. Annals of the New York Academy of Sciences 8r, 950-966.

Stauffer, J. F. \& Backus, M. P. (1954). Spontaneous and induced variation in selected stocks of the Penicillium chrysogenum series. Annals of the New York Academy of Sciences 60, art. I. 